\title{
Nitric Oxide Synthase Inhibitors Preclinical Studies of Potential Use for Treatment of Opioid Withdrawal
}

\author{
D. Bruce Vaupel, Ph.D., Alane S. Kimes, Ph.D., and Edythe D. London, Ph.D.
}

Four inhibitors of nitric oxide synthase (NOS), administered as acute pretreatments, attenuated several signs of naloxone-precipitated opioid withdrawal in morphine-dependent rats. Profiles of these drugs for inhibiting the expression of withdrawal were similar to that of clonidine, a drug used clinically to treat opioid withdrawal. The nonselective NOS inhibitors, $N^{G}$-nitro$\mathrm{L}$-arginine and $N^{G}$-nitro-L-arginine methyl ester, and $N(5)$-(1-iminoethyl)-L-ornithine, a selective inhibitor of endothelial NOS, increased blood pressure in awake, morphine-naive and morphine-dependent rats not

KEY WORDS: Nitric oxide; Nitric oxide synthase inhibitors; Opioid withdrawal; Opiate withdrawal; Morphine; Hypertension; Rats

The phenomena of opioid tolerance and dependence involve a variety of nonopioid systems, including adrenergic and cholinergic neurotransmission. Among the newest candidates to receive attention as a potential endogenous modulator of the long-term effects of opioids is nitric oxide. This freely diffusible gas is formed in an enzymatic reaction catalyzed by nitric oxide synthase (NOS).

Nitric oxide synthase is among the largest and most

From the Neuroimaging and Drug Action Section, Intramural Research Program, National Institute on Drug Abuse, National Institutes of Health (DBV, ASK, EDL); Department of Radiology, Johns Hopkins Medical Institutions; and Department of Pharmacology and Experimental Therapeutics, School of Medicine, University of Maryland, Baltimore, MD (EDL).

Address correspondence to: D. Bruce Vaupel, Ph.D., Neuroimag ing and Drug Action Section, NIDA Division of Intramural Research P.O. Box 5180, Baltimore, MD 21224

Received December 15, 1994; revised Iune 14, 1995; accepted June 19, 1995. undergoing withdrawal. 7-Nitroindazole, a selective inhibitor of neuronal NOS, did not elevate blood pressure. Insofar as hypertension is a component of opioid withdrawal in humans, the ability of 7-nitroindazole to attenuate morphine withdrawal in rats without eliciting a vasopressor response suggests that 7-nitroindazole may have human therapeutic potential. Research directions for the continued development of 7-nitroindazole as a therapeutic modality are discussed with respect to issues of physical dependence, tolerance, and safety.

[Neuropsychopharmacology 13:315-322, 1995]

complicated of enzymes (Nathan and Xie 1994), and as many as six to eight isoforms of NOS (Nathan and Xie 1994; Murad 1994) have been identified from neurons, macrophages, and endothelial cells. These isoforms are classified as either constitutive or inducible. Constitutive isoforms are regulated by $\mathrm{Ca}^{2+}$ and calmodulin, and represent a low output pathway for the production of nitric oxide involved in homeostasis. Inducible types of NOS are activated by endotoxin and some cytokines, and this induction is considered a high output mechanism to produce nitric oxide for certain functions, such as host defense.

In its role as a neuromodulator, nitric oxide produced by constitutive neuronal NOS has been linked to the N-methyl-D-aspartate (NMDA) receptor complex. Nitric oxide production by this enzyme is initiated by activation of the NMDA receptor-gated ion channel, a step that enhances influx of $\mathrm{Ca}^{2+}$ into the cell. $\mathrm{Ca}^{2+}$ then complexes with calmodulin, which in turn binds to NOS, thereby activating the enzyme. The activated NOS may then convert the substrate, L-arginine, to nitric oxide and L-citrulline.

Interest in the role of nitric oxide in opioid with- 
drawal was prompted by investigations demonstrating that treatments known to inactivate the NMDA receptor diminished signs of morphine withdrawal. Kynurenic acid, a nonselective antagonist of excitatory amino acid receptors, suppressed morphine withdrawal (Rasmussen et al. 1991b). In addition, MK-801 (dizocilpine), a noncompetitive antagonist of the NMDA receptor and LY274614, a competitive NMDA receptor antagonist, reduced morphine withdrawal (Rasmussen et al. 1991a; Trujillo and Akil 1991a). Concerns about the phencyclidinelike side-effects and possible neurotoxicity of MK-801 (Olney et al. 1989) generated reservations about the potential clinical utility of this drug. In light of the relation between NMDA receptor activation and nitric oxide production, observations that both MK-801 and LY274614, which are antagonists of NMDA receptor activation, reduced withdrawal suggested an association between nitric oxide production and opioid dependence.

To test this hypothesis, we manipulated the production of nitric oxide at the level of NOS and focused on the ability of four NOS inhibitors to modify morphine withdrawal in rats. In additional studies, we evaluated the cardiovascular effects of these NOS inhibitors in morphine-naive and morphine-dependent states (Vaupel et al. 1995). Initially, $N^{G}$-nitro-L-arginine (L-NNA) and $\mathrm{N}^{\mathrm{G}}$-nitro-L-arginine methyl ester (L-NAME), two derivatives of $\mathrm{L}$-arginine with modified guanido groups (Figure 1), were tested. Both nitro derivatives are nonselective inhibitors of NOS and produce a characteristic increase in blood pressure (Dir. nagl et al. 1993; Rees et al. 1990). Parallel to the differentiation of the isoforms of NOS, two isoformselective NOS inhibitors have been developed. N(5)(1-iminoethyl)-L-ornithine (L-NIO), a highly potent inhibitor of endothelial NOS (Rees et al. 1990), elevates blood pressure. 7-Nitroindazole (7-NI), a fused heterocyclic compound, is unique because it selectively inhibits murine neuronal NOS but does not increase mean arterial pressure (MAP) in anesthetized mice (Moore et al. 1993).

\section{ATTENUATION OF OPIOID WITHDRAWAL}

\section{Production of Physical Dependence on Morphine and Precipitation of Withdrawal}

Physical dependence was produced in male, Fischer344 rats by subcutaneously implanting 75 -mg morphine pellets using one of two dosing schedules. For our initial studies, morphine dependence was produced by implanting one pellet on day 1 and two additional pellets on day 4 . Withdrawal was precipitated on day 8 using naloxone. For the second group of experiments, a lower level of dependence was produced. One pellet was implanted on day 1 , and naloxone-precipitated<smiles>C=C(N)NCCC[C@H](N)C(=O)O</smiles><smiles>CC(=N)NCCC[C@H](N)C(=O)O</smiles>

N(5)-(1-iminoethyl)-L-ornithine<smiles>N#CC(=O)OC(N)CCCNC(N)=NN=O</smiles><smiles>O=[N+]([O-])c1cccc2cn[nH]c12</smiles>

7-nitroindazole<smiles>COC(=O)CCCNC(N)=NN=O</smiles>

\section{NG-nitro-L-arginine methyl ester}

Figure 1. Structures of four inhibitors of nitric oxide synthase evaluated for their ability to reduce signs of naloxoneprecipitated morphine withdrawal are illustrated along with the natural enzyme substrate, L-arginine, which is converted to nitric oxide.

withdrawal tests were conducted on day 4 . In all experiments, withdrawal was precipitated with naloxone $(0.5 \mathrm{mg} / \mathrm{kg}, \mathrm{SC})$, and 18 signs of morphine withdrawal were measured and scored over 15 minutes by one observer who was blinded to the treatment conditions according to published procedures (Kimes et al. 1993).

\section{Attenuation of Opioid Withdrawal by L-NNA}

In initial experiments, L-NNA was tested in rats made dependent on morphine (three pellets) over an 8-day period. L-NNA was administered in doses of 1,3 , or $7.5 \mathrm{mg} / \mathrm{kg}$ IP b.i.d. on days 4 to 7 of morphine treatment. The dosing schedule was based on a report indicating that 4 days of treatment with L-NNA produced maximum enzyme inhibition. In placebo-pelleted animals, naloxone administered following treatment with $7.5 \mathrm{mg} / \mathrm{kg}$ of L-NNA for 4 days produced no withdrawal signs. Morphine-pelleted rats receiving naloxone alone exhibited signs of opioid withdrawal, and L-NNA pretreatment reduced naloxone-precipitated weight loss $(1,3$, and $7.5 \mathrm{mg} / \mathrm{kg}, p<.05)$ and wet dog shakes $(7.5 \mathrm{mg} / \mathrm{kg}, p<.05)$, but these effects were not linearly related to the dose of L-NNA (Kimes et al. 1993). 
The absence of dose-dependent decreases suggested that the 4-day dosing regimen produced maximal enzyme inhibition by all three doses of L-NNA.

\section{Effect of the Dosing Schedule on Attenuating Morphine Withdrawal}

Additional experiments were performed to determine whether it was necessary to coadminister the NOS inhibitor or if a single pretreatment would be effective in reducing withdrawal signs. Consequently, the time course of the L-NNA treatment was varied. Morphinedependent rats (three-pellet schedule) were subjected to one of three L-NNA dosing regimens, as follows: (1) saline or $7.5 \mathrm{mg} / \mathrm{kg} \mathrm{L-NNA} \mathrm{IP} \mathrm{b.i.d.} \mathrm{on} \mathrm{days} 4$ to 7 with withdrawal precipitated on day 8 as in the first experiment; (2) saline or $7.5 \mathrm{mg} / \mathrm{kg}$ L-NNA IP, 1 hour before naloxone; or (3) saline or $15 \mathrm{mg} / \mathrm{kg} /$ day L-NNA for 8 days delivered by Alzet minipumps implanted on day 1.

The effects of varying treatment durations were more prominent on tests of the suppression of wet dog shakes and weight loss than on antagonizing other signs (Kimes et al. 1993). Wet dog shakes were significantly reduced by all three dosing schedules; however, weight loss was not uniformly affected. Reductions in weight loss were produced by the acute and 4-day treatments with L-NNA, but not by the 8-day infusion. Based on the effectiveness of the 1-hour L-NNA pretreatment. we concluded that acutely inhibiting NOS reduces the expression of some components of the opioid withdrawal syndrome.

We next demonstrated that a 1-hour pretreatment with doses of L-NNA $(0.3,1,3$, or $10 \mathrm{mg} / \mathrm{kg})$ and L-NAME $(7.5,15,30$, or $60 \mathrm{mg} / \mathrm{kg})$ produced significant $(p<.05)$, dose-dependent decreases in wet dog shakes and weight loss and an increase in teeth chattering. However, we questioned why only these particular signs were affected. For this reason, the effectiveness of NOS inhibitors to attenuate opioid withdrawal using a lower level of morphine dependence was tested. Because the $\alpha_{2}$-adrenoceptor agonist clonidine is a treat ment for opioid withdrawal, it was included as a posi tive, active control in this study.

\section{Dose-Response and Efficacy}

The dose-response relationships of four NOS inhibitors (Figure 1) and clonidine were compared in rats made morphine-dependent using one 75-mg morphine pellet. A short time-course study demonstrated that peak withdrawal occurred on day 4 after the implantation of one pellet (day 1), and all subsequent precipitation experiments were conducted using this protocol. NOS inhibitors and clonidine were injected IP 1 hour before naloxone-precipitated withdrawal. The studies were performed in four blocks of experiments, and pretreatments were randomized within blocks. Saline and other appropriate vehicle controls were included in each block.

7-NI, L-NAME, L-NNA, and L-NIO produced significant, dose-dependent decreases in weight loss, diarrhea, wet dog shakes, and grooming behavior, and collectively they did not affect abnormal posturing and ptosis (Vaupel et al. 1995). These data are summarized in Table 1, and the dose-response curves for wet dog shakes and weight loss are shown in Figure 2. Other withdrawal behaviors were consistently affected by three of four NOS inhibitors. L-NAME, L-NNA and $\mathrm{L}-\mathrm{NIO}$ increased the incidence of penis licks and ejaculations as indicated by a significant treatment effect in a one-way analysis of variance (ANOVA); these three inhibitors did not affect mastication. In contrast, 7-NI exhibited a trend to decrease genital effects (one-way ANOVA, treatment effect, NS) and significantly inhibited mastication (Table 1). 7-NI, L-NNA, and L-NIO linearly increased exploratory activity. The most variable withdrawal signs were escape jumps and salivation. Only 7-NI and L-NIO increased escape jumps, and no consistent pattern developed for effects on salivation. There were no differences between saline responses and those produced by the peanut oil vehicle used for 7-NI or the acidified saline vehicle used for L.-NNA.

From these data, profiles for modifying morphine withdrawal were established for each NOS inhibitor (Table 1) for comparison with the effects of clonidine. Clonidine reduced weight loss, diarrhea, and wet dog shakes, as did the four NOS inhibitors. Clonidine also decreased abnormal posturing and ptosis-signs that were not significantly modified by any NOS inhibitorand increased some withdrawal signs, particularly exploratory activity and escape jumps. Genital-related activity was increased with clonidine, but not to a significant degree. Based upon modifications of the naloxone-precipitated morphine withdrawal syndrome, the effects of clonidine were most similar to those of 7-NI and L-NIO (Table 1) (Vaupel et al. 1995).

\section{Relative Potency Determinations}

Potency estimates for the NOS inhibitors, which were based upon attenuation of weight loss and wet dog shakes, were computed using the parallel line bioassay statistical model (Figure 2). In order to make potency comparisons, this model requires pairs of doseresponse curves to be parallel, to exhibit linear regression, and to encompass comparable portions of their respective dose-response curves. Overall, the range of potency estimates for the NOS inhibitors was not very large; for antagonism of weight loss, relative potencies 
Table 1. Effects of NOS Inhibitors and Clonidine on Naloxone-Precipitated Opioid Withdrawal, Mean Arterial Blood Pressure, and Heart Rate

\begin{tabular}{|c|c|c|c|c|c|}
\hline & 7-NI & L-NIO & L-NAME & L-NNA & Clonidine \\
\hline \multicolumn{6}{|c|}{$\begin{array}{l}\text { Effects of NOS inhibitors and } \\
\text { clonidine on naloxone- } \\
\text { precipitated } \\
\text { opiate withdrawal }\end{array}$} \\
\hline Weight loss & $\downarrow$ & $\downarrow$ & $\downarrow$ & $\downarrow$ & $\downarrow$ \\
\hline Diarrhea & $\downarrow$ & $\downarrow$ & $\downarrow$ & $\downarrow$ & $\downarrow$ \\
\hline Wet dog shakes & $\downarrow$ & $\downarrow$ & $\downarrow$ & $\downarrow$ & $\downarrow$ \\
\hline Grooming & $\downarrow$ & $\downarrow$ & $\downarrow$ & $\downarrow$ & $\uparrow$ \\
\hline Mastication & $\downarrow$ & $\varnothing$ & $\varnothing$ & $\varnothing$ & $\downarrow N R$ \\
\hline Salivation & $\downarrow$ & $\uparrow$ & $\varnothing$ & $\varnothing$ & $\varnothing$ \\
\hline Penis licks/ejaculation & $\downarrow Q$ & $\uparrow$ & $\uparrow$ & $\uparrow$ & $\uparrow \quad Q$ \\
\hline Exploratory activity & $\uparrow$ & $\uparrow$ & $\varnothing$ & $\uparrow$ & $\uparrow$ \\
\hline Escape jumps & $\uparrow$ & $\uparrow$ & $\varnothing$ & $\varnothing$ & $\uparrow$ \\
\hline Abnormal posturing & $\varnothing$ & $\varnothing$ & $\varnothing$ & $\varnothing$ & $\downarrow$ \\
\hline Ptosis & $\varnothing$ & $\varnothing$ & $\varnothing$ & $\varnothing$ & $\downarrow N R$ \\
\hline \multicolumn{6}{|c|}{$\begin{array}{l}\text { Cardiovascular effects of NOS } \\
\text { inhibitors and clonidine }\end{array}$} \\
\hline MAP, naive & $\varnothing$ & $\uparrow$ & $\uparrow$ & $\uparrow$ & $\downarrow \quad \mathrm{H}$ \\
\hline MAP, dependent & $\tilde{\varnothing}$ & $\uparrow$ & $\uparrow$ & $\uparrow$ & $\downarrow \mathrm{H}$ \\
\hline Heart rate, naive & $\varnothing$ & $\downarrow$ & $\downarrow$ & $\downarrow$ & - \\
\hline Heart rate, dependent & $\downarrow$ & $\downarrow$ & $\downarrow$ & $\downarrow$ & - \\
\hline
\end{tabular}

A $\downarrow$ or $\uparrow$ indicates a decrease or increase, respectively, as determined by a significant treatment effect and a $\log$-linear regression; $\varnothing=$ no effect; $N R=$ no regression, but a significant treatment effect (oneway ANOVA, $p<.05$ ); $Q=$ a qualitative effect (i.e., treatment effect did not attain statistical significance); naive and dependent refer to morphine-naive and morphine-dependent rats, respectivelv: $\mathrm{H}=$ clinical effect.

ranged from 0.4 to 4.0 . The potency estimates for diarrhea were similar to those for weight loss (data not shown). For wet dog shakes, L-NNA was equipotent to L-NAME because the confidence limits included 1.0, and 7-NI was estimated to be 0.4 times as potent as L-NAME. Potency estimates for clonidine were not determined, but based on the effectiveness of the doses tested $(0.01$ to $0.1 \mathrm{mg} / \mathrm{kg})$, clonidine was at least an order of magnitude more potent than L-NNA, the most potent NOS inhibitor.

\section{ADVERSE REACTIONS}

\section{Behavioral Toxicity and Lethality}

Whereas all four NOS inhibitors and clonidine modified certain signs of opioid withdrawal in rats, they also produced some behavioral toxicity and lethality. Ataxia was produced by $0.1 \mathrm{mg} / \mathrm{kg}$ clonidine in six of eight rats and by $100 \mathrm{mg} / \mathrm{kg} 7-\mathrm{NI}$ in eight of eight rats. These dosages of each drug were the highest tested. Clonidine also produced catalepsy in three of eight rats at $0.1 \mathrm{mg} / \mathrm{kg}$. Observations of the animals for 3 days after precipitating withdrawal revealed no deaths followed the administration of 7-NI, L-NIO, or clonidine, but some animals treated with L-NNA (four of eight receiving 30 $\mathrm{mg} / \mathrm{kg}$ ) and L-NAME (two of 16 at $30 \mathrm{mg} / \mathrm{kg}$ and one of 16 at $56 \mathrm{mg} / \mathrm{kg}$ ) died.

\section{Cardiovascular Effects of NOS Inhibitors}

Behavioral changes observed in human subjects have not been the sole determinant of withdrawal intensity. Himmelsbach incorporated an increase in blood pressure as an index of autonomic hyperactivity into his scoring system of evaluating withdrawal in humans (Himmelsbach 1937). Increases in arterial blood pressure also are a reliable, objective measure of both withdrawal intensity and the degree of physical dependence in the rat morphine model (Marshall and Buccafusco 1985). Consequently, adjunctive treatment of opioid withdrawal with an NOS inhibitor could result in an additive or potentiated vasopressor response as NOS inhibitors, such as L-NAME or L-NNA, elevate blood pressure in rats not subjected to opioid withdrawal. The lack of vasopressor activity by 7-NI makes this NOS inhibitor an especially attractive candidate for evaluation in morphine-dependent animals. An initial experiment was designed to answer the following two questions: (1) Does 7-NI lack vasopressor activity in unanesthetized rats?, (2) Are the cardiovascular responses to 7-NI similar in morphine-naive and in morphinedependent rats?

Blood pressure experiments used rats that were partially immobilized and had their left femoral artery and vein catheterized under halothane anesthesia (London et al. 1981). Studies, conducted 3 hours after recovery from anesthesia, were performed using a pressure 


\section{Weight Loss}

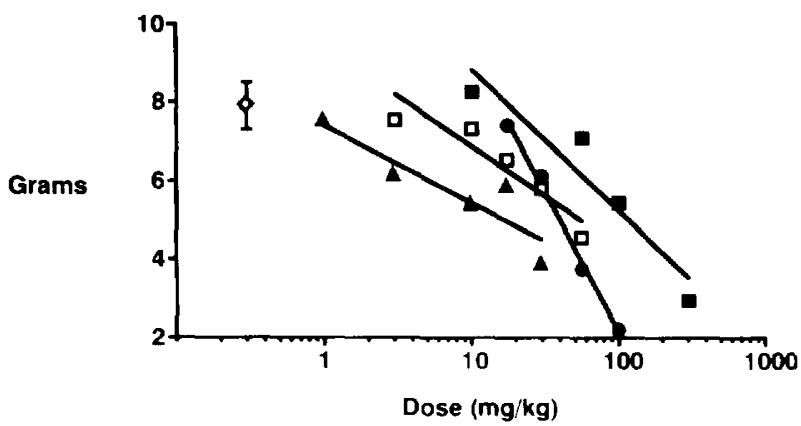

\section{Wet Dog Shakes}

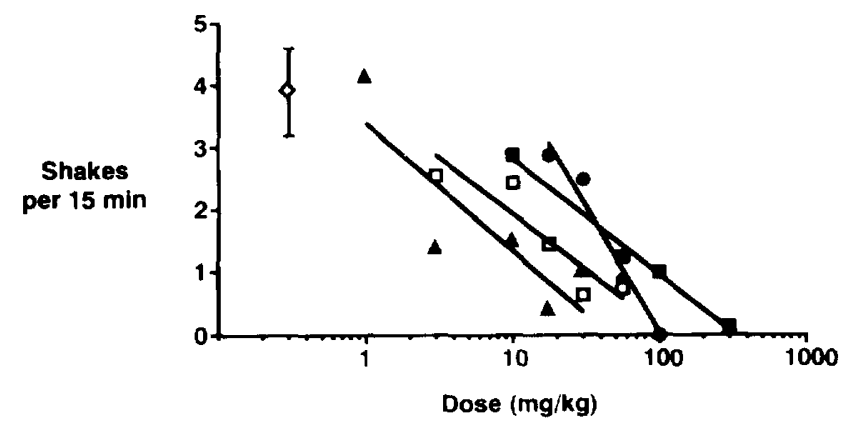

Figure 2. Relative potency determinations for nitric oxide synthase inhibitors based on ability to reduce weight loss and wet dog shakes produced by naloxone-precipitated morphine withdrawal. L-NAME was designated as the standard drug and its potency was set equal to 1.0. Relative potency estimates, with their $95 \%$ confidence limits shown within parentheses, are shown next to the abbreviations. Each point represents the mean of 8 to 16 animals. The saline response represents the mean \pm SEM. The weight loss dose-response curve for 7-NI (top panel) was not parallel to the L-NAME curve and, therefore, no potency estimate could be made. Although the 7-NI curve for wet dog shakes tended to have a relatively steeper slope than that of L-NAME (bottom panel), the slopes were not statistically significant. RP $(95 \%$ CL); $\square$ L-NAME 1.0; $\Delta$ L-NNA 4.4 (2.1-10.6) (top panel) and 2.0(0.7-5.2) (bottom panel); L-NIO 0.4 (0.2-0.7); 7-NI not parallel (top panel) and $0.4(0.2-0.9)$ (bottom panel); 0 saline (control)

transducer and chart recorder to determine systolic, diastolic, and mean arterial pressures (MAP) and heart rate. After obtaining baseline measurements, either drug or vehicle was injected IP, and cardiovascular effects were recorded over 90 minutes. Identical experiments were run in naive and morphine-dependent rats (tested on day 4 after receiving a single 75 -mg morphine pellet).

Vasopressor effects of the NOS inhibitors are presented in Table 2 . The doses tested represented the highest dose of each NOS inhibitor used to attenuate withdrawal (Table 2). Of the four NOS inhibitors administered to morphine-naive and morphine-dependent rats, only 7-NI failed to increase MAP. Generally, maximal increases in MAP developed approximately 1 hour after administration, and MAP remained relatively stable in animals given L-NIO, L-NAME, or LNNA. Neither saline nor peanut oil affected MAP. At 60 minutes after drug administration, the time corresponding to naloxone administration in the precipitated abstinence experiments, the differences in MAP from baseline values approximated $40 \mathrm{~mm} \mathrm{Hg}$ in both the morphine-naive and -dependent rats given L-NIO, LNAME, or L-NNA. The failure of $7-\mathrm{NI}$ to elicit a vasopressor response, although having a significant effect on opioid withdrawal, suggests that inhibiting endothelial NOS is not necessary to attenuate withdrawal.

NOS inhibitors, which increased MAP, also produced decreases in heart rate that were nearly maximal 1 hour after treatment. Although one might hypothesize that the bradycardia was a vasovagal reflex response, results with $7-\mathrm{NI}$ argue against this view. In morphine-naive rats, 7-NI tended to slow heart rate, even though the drug lacked vasopressor activity. This bradycardia was greater in morphine-dependent rats and represented a significant decrease comparable to the reductions produced by the other NOS inhibitors. Therefore, the bradycardia induced by 7-NI and the other NOS inhibitors as well must, at least to some extent, reflect a direct action, presumably of central origin.

\section{DISCUSSION}

\section{Role for Nitric Oxide in Withdrawal}

Since the first report that L-NNA inhibits opioid withdrawal (Kimes et al. 1991), we have expanded our assessment of NOS inhibitors. Four NOS inhibitors have now been shown consistently to reduce several signs characteristic of the naloxone-precipitated abstinence syndrome in morphine-dependent rats in a dosedependent manner. These inhibitors also increased a few signs. Similar spectra of activity were produced by both the nonselective (L-NNA and L-NAME) and selective (7-NI and L-NIO) NOS inhibitors. Other investigators also have reported inhibition of morphine abstinence in Sprague-Dawley rats and Swiss mice (Adams et al. 1993; Cappendijk et al. 1993) by acutely administered L-NAME and L-NNA. Thus, three laboratories have concluded that NOS inhibitors are capable of reducing the expression of morphine withdrawal. Conversely, increasing levels of nitric oxide by administering isosorbide dinitrate, a nitric oxide donor, induces a quasi-morphine-abstinence syndrome in naive rats (Adams et al. 1993). Collectively, these data 
Table 2. Cardiovascular Responses to NOS Inhibitors

\begin{tabular}{|c|c|c|c|c|}
\hline & \multicolumn{2}{|c|}{$\begin{array}{l}\text { Mean Arterial Pressure } \\
\text { (change in } \mathrm{mm} \mathrm{Hg} \text { ) }\end{array}$} & \multicolumn{2}{|c|}{$\begin{array}{l}\text { Heart Rate } \\
\text { (change in beats/minute) }\end{array}$} \\
\hline & Morphine-naive & Morphine-dependent & Morphine-naive & Morphine-dependent \\
\hline Saline & $3 \pm 7$ & $0 \pm 5$ & $-13 \pm 26$ & $-2 \pm 12$ \\
\hline Peanut oil & $1 \pm 6$ & $0 \pm 1$ & $-10 \pm 20$ & $10 \pm 6$ \\
\hline $7-\mathrm{NI}(100 \mathrm{mg} / \mathrm{kg})$ & $3 \pm 10$ & $-10 \pm 5$ & $-67 \pm 32$ & $-160 \pm 15^{a}$ \\
\hline L-NAME $(56 \mathrm{mg} / \mathrm{kg})$ & $40 \pm 9^{a}$ & $43 \pm 7^{a}$ & $-130 \pm 26^{b}$ & $-107 \pm 32^{a}$ \\
\hline L-NNA $(30 \mathrm{mg} / \mathrm{kg})$ & $45 \pm 9^{a}$ & $49 \pm 10^{a}$ & $-163 \pm 15^{a}$ & $-146 \pm 23^{a}$ \\
\hline $\mathrm{L}-\mathrm{NIO}(300 \mathrm{mg} / \mathrm{kg})$ & $48 \pm 3^{a}$ & $41 \pm 1^{a}$ & $-123 \pm 22^{h}$ & $-136 \pm 20^{a}$ \\
\hline
\end{tabular}

Responses shown were measured in unanesthetized naive and morphine-dependent rats. Morphine dependence was produced by implanting one 75-mg morphine pellet. Values (mean \pm SEM) represent the change from baseline occurring at 60 minutes after IP treatment with a vehicle or nitric oxide synthase inhibitor. This time corresponds to the pretreatment interval in the naloxone-precipitated morphine withdrawal experiments. Peanut oil was the vehicle for 7-NI. Otherwise, saline was the vehicle. Responses were based on an $n$ of 3 except for saline for which $n=6$. Drug effects that were significantly different from the effects of saline are indicated as ${ }^{a} p<.01$ or ${ }^{b} p<.05$ (Dunnett's test). There was a small statistical difference in the average $(n=24) \mathrm{MAP}$ baseline values for the naive $(128 \pm 1 \mathrm{~mm} \mathrm{Hg})$ and morphine-dependent groups $(125 \pm 1 \mathrm{~mm} \mathrm{Hg})(p<.02)$, although the physiologic significance of this difference is debatable. Average heart rates were equivalent for the naive $(434 \pm 8$ beats $\mathrm{min})$ and morphine-dependent $(433 \pm 9$ beats/min) groups.

support the concept that nitric oxide partially mediates the morphine withdrawal syndrome.

In the course of establishing profiles for attenuating morphine withdrawal, a degree of variability was observed including the finding that some withdrawal signs were increased. This variability was not surprising as increases in some withdrawal signs have been reported with clonidine treatment (Kimes et al. 1990; Tseng et al. 1975). Part of the variability among four NOS inhibitors may be attributable to the relatively low level of dependence used in our comparison study, that 7-NI was a specific inhibitor of neuronal NOS, and lastly that nitric oxide-mediated mechanisms have a subsidiary rather than primary role in opioid withdrawal.

The development of opioid tolerance and dependence are temporally associated. Thus, it is not surprising that opioid tolerance as well as dependence appear to be modulated by nitric oxide. Investigations conducted by Kolesnikov et al. $(1992,1993)$ evaluated the effect of L-NNA on tolerance to the antinociceptive actions of morphine. They found that L-NNA effectively blocked the development of tolerance and was capable of reversing pre-existing tolerance.

The relative extent to which central and peripheral inhibition of NOS contributes to the attenuation of opioid withdrawal as well as the relevant neuroanatomic sites of action and the associated pathways remain unknown. NOS cloned from brain appears to be exclusively neuronal or endothelial (Dawson et al. 1992). Further, a single dose of $7-\mathrm{NI}(30 \mathrm{mg} / \mathrm{kg}$ IP) given to rats produces $80 \%$ to $85 \%$ inhibition of NOS measured ex vivo in the striatum, hippocampus, cerebral cortex, olfactory bulb, and cerebellum (MacKenzie et al. 1994). The identification of NOS-containing neurons in the spinal cord implies roles for nitric oxide in preganglionic sympathetic and parasympathetic, somatosensory, and visceral pathways (Saito et al. 1994). Based on this in- formation, we conclude that the attenuation of opioid withdrawal by 7-NI, and the other NOS inhibitors as well, is mediated substantially by a central component of action. Stereospecific reversal of the effect of a NOS inhibitor effect would constitute strong evidence for the involvement of NOS, but presently whether or not there is stereoselective antagonism by arginine of the attenuating effects of NOS inhibitors on withdrawal is a critical, unanswered question. Future experiments geared at elucidating involvement of NOS in opioid actions will benefit from new research tools, such as isoform-specific NOS antibodies and genetically altered mice lacking neuronal NOS (Huang et al. 1993), especially if they are used in conjunction with established techniques such as measurement of opioid withdrawal. For example, mice lacking neuronal NOS could be used to determine to what degree the attenuation of withdrawal produced by NOS inhibitors is independent of neuronal NOS. Equally important would be studies of whether the absence of neuronal NOS alters the development of physical dependence or tolerance to morphine.

The mechanism by which NOS inhibitors attenuate withdrawal is unclear. It is not known to what extent these drugs prevent the development of morphine dependence, reverse existing dependence, or block the expression of withdrawal by acting at sites downstream from those that participate in the production and maintenance of morphine dependence. Kolesnikov et al. (1993) reported that chronically dosing mice with L-NNA concomitantly with morphine prevented precipitated withdrawal, implying an effect on the development of dependence. In other experiments, the effect of daily administration of L-NNA initiated on the sixth day of a 10-day morphine dosing schedule was interpreted as reversing dependence (Kolesnikov et al. 1993). In our studies, in which L-NNA was equally as effec- 
tive in reducing withdrawal signs when the drug was given 1 hour before precipitation of withdrawal as when given over the last 4 of 7 days of morphine administration, it is clear that the action primarily represents antagonism of the expression of withdrawal and not blockade of the development of dependence. Nonetheless, even in our studies, L-NNA may have either antagonized the expression of withdrawal or reversed previously established dependence without acting directly on the central substrates of opioid dependence (Kimes et al. 1993).

\section{Efficacy of NOS Inhibitors and Clonidine}

The profiles of the four NOS inhibitors that modify morphine withdrawal were developed and compared specifically to that of clonidine, which is used clinically to alleviate opioid withdrawal. In general, the attenuating effects of clonidine were similar, but not identical, to those produced by the NOS inhibitors. Furthermore, in addition to reducing numerous signs of withdrawal, both clonidine and the NOS inhibitors enhanced a small number of withdrawal signs. In view of the similar spectra of effects of the four NOS inhibitors evaluated and clonidine on morphine withdrawal signs and the clinical utility of clonidine, this similarity suggests that NOS inhibitors have the potential to be effective therapeutic agents in treating opioid withdrawal, provided other pharmacologic issues are addressed.

Insofar as relatively high doses of the NOS inhibitors were necessary to effectively suppress signs of morphine withdrawal, the involvement of other mechanisms cannot be discounted. As deaths occurred after the administration of L-NNA and L-NAME in the present study, nonspecific effects may be more of a concern for these inhibitors than for 7-NI and L-NIO. Another laboratory has reported that L-NAME and L-NNA reduced naloxone-precipitated withdrawal jumping in mice, but the highest doses exacerbated withdrawal and inverted the dose-response curve suggesting involvement of nonspecific or additional mechanisms (Cappendijk et al. 1993). Such an inflection could result from antimuscarinic activity associated with L-NAME (Buxton et al. 1993). We observed no U-shaped doseresponse curves. However, using smaller doses does not necessarily simplify data interpretation. For example, Rasmussen et al. (1991a, 1991b) reported that the noncompetitive NMDA antagonist MK-801 attenuated opiate withdrawal at relatively low dose levels $(0.1$ to $0.5 \mathrm{mg} / \mathrm{kg}$, IP), yet it concomitantly produced PCPlike behaviors. In comparison, higher doses of the competitive NMDA antagonist LY274614 (25 to $100 \mathrm{mg} / \mathrm{kg}$, IP) suppressed morphine withdrawal but produced no PCPlike actions, yet both LY274614 and MK-801 may indirectly reduce NOS activity. Nevertheless, certain nonspecific influences may be discounted. Our results demonstrated that the vasopressor effects of NOS inhibitors are not responsible for attenuating opioid withdrawal. Production of ataxia also has the potential to nonspecifically inhibit motoric signs of withdrawal. Notwithstanding, both clonidine and 7-NI, despite producing ataxia, increased exploratory activity and escape jumps. Because compounds such as 7-NI and L-NIO are relatively new pharmaceuticals, their doserelated interactions with other neurochemical systems and drugs remain to be defined.

Identification of behavioral toxicities associated with the administration of NOS inhibitors to rats raises the issue of the margin of safety. The sedative properties of clonidine in both animals and humans are well known, but they have not been sufficient to prevent the use of clonidine as a therapeutic agent. This issue is relevant as MK-801, which attenuated signs of opioid withdrawal presumably by reducing levels of nitric oxide, enhanced the cataleptic and lethal effects of morphine (Trujillo and Akil 1991b). Thus, there is a need to determine measures of safety, such as therapeutic indices, for NOS inhibitors, especially 7-NI.

Prior to the development of 7-NI, the potential clinical use of any nonselective NOS inhibitor in treating opioid withdrawal would be eliminated, in all likelihood, by its prominent effect on blood pressure. Other issues aside, the failure of 7-NI to increase blood pressure in naive and morphine-dependent animals makes it the only viable candidate, in our opinion, among the four NOS inhibitors tested for potential therapeutic development for treating opioid withdrawal. What remains to be assessed is a determination of the dynamic blood pressure effects occurring during withdrawal after pretreatment with an NOS inhibitor. Experience gained from the rat model of opioid withdrawal suggests that the therapeutic promise of additional NOS inhibitors targeted for specific disease states will likely reside in the discovery of isoform-selective inhibitors of NOS.

\section{SUMMARY}

The arginine-NOS-nitric oxide pathway participates in the mediation of naloxone-precipitated opioid withdrawal. A modulatory role for nitric oxide is supported by the ability of four NOS inhibitors to partially attenuate characteristic signs of the morphine abstinence syndrome in the rat. Additional evidence has linked NOS with the development of opioid tolerance. Thus, two lines of pharmacologic evidence have demonstrated a role for nitric oxide in opioid tolerance and dependence and suggest that future studies continue to explore the potential clinical applications of NOS inhibitors in the areas associated with the development of tolerance to 
the analgesic effects of opiates and amelioration of the opioid withdrawal syndrome.

\section{ACKNOWLEDGMENTS}

The authors thank Veronica Anne Williams for technical assistance and Cindy Ambriz for preparing the manuscript.

\section{REFERENCES}

Adams ML, Kalicki JM, Meyer ER, Cicero TJ (1993): Inhibition of the morphine withdrawal syndrome by a nitric oxide synthase inhibitor, $\mathrm{N}^{\mathrm{C}}$-nitro-L-arginine methyl ester. Life Sci 52:PL245-PL249

Buxton ILO, Cheek DJ, Eckman D, Westfall DP, Sanders KM, Keef KD (1993): $N^{G}$-Nitro L-arginine methyl ester and other alkyl esters of arginine are muscarinic receptor antagonists. Circ Res 72:387-395

Cappendijk SL, de Vries R, Dzoljic MR (1993): Inhibitory effect of nitric oxide (NO) synthase inhibitors on naloxoneprecipitated withdrawal syndrome in morphine-dependent mice. Neurosci Lett 162:97-100

Dawson TM, Dawson VL. Snyder SH (1992): A novel neuronal messenger molecule in brain: The free radical, nitric oxide. Ann Neurol 32:297-311

Dirnagl U, Lindauer U, Villringer A (1993): Role of nitric uxide in the coupling of cerebral blood flow to neuronal ativation in rats. Neurosci Lett 149:43-46

Himmelsbach CK (1937): Clinical studies of drug addiction. II. "Rosium" treatment of drug addiction. Public Health Rep Suppl 125:1-18

Huang PL, Dawson TM, Bredt DS, Snvder SH, Fishman MC (1993): Targeted disruption of the neuronal nitric oxide synthase gene. Cell 75:1273-1286

Kimes AS, Bell JA, London ED (1990): Clonidine attenuates increased brain glucose metabolism during naloxoneprecipitated morphine withdrawal. Neuroscience 34 633-644

Kimes AS, Vaupel DB, Bruckner M, London ED (1991): Nitroarginine, a nitric oxide svnthase inhibitor, attenuates morphine withdrawal. Soc Neurosci Abstr 17:538

Kimes AS, Vaupel DB, London ED (1993): Attenuation of some signs of opioid withdrawal by inhibitors of nitric oxide synthase. Psychopharmacology (Berl) 112:521-524

Kolesnikov YA, Pick CG, Pasternak GW (1992): $N^{(-n i t r o-}$ 1.-arginine prevents morphine tolerance. Eur J Pharmacol 221:399-400

Kolesnikov YA, Pick C C, Ciszew ska G, Pasternak GW (1943) Blockade of tolerance to morphine but not to $k$ opioids by a nitric oxide synthase inhibitor. Proc Natl Acad Sci USA 90:5162-5166

London ED, Nespor SM, Ohata M, Rapoport SI (1981): Local cerebral glucose utilization during development and aging of the Fischer-344 rat. J Neurochem 37:217-221

MacKenzie GM, Rose S, Bland-Ward PA, Moore PK, Jenner $P$, Marsden CD (1994): Time course of inhibition of brain nitric oxide synthase by 7 -nitro indazole. NeuroReport 5:1993-1996

Marshall DC, Buccafusco JJ (1985): Supraspinal and spinal mediation of naloxone-induced morphine withdrawal in rats. Brain Res 329:131-142

Moore PK, Babbedge RC, Wallace P, Gaffen ZA, Hart SL (1993): 7-Nitro indazole, an inhibitor of nitric oxide synthase, exhibits antinociceptive activity in the mouse without increasing blood pressure. Br J Pharmacol 108:296-297

Murad F (1994): The role of nitric oxide in modulating guanylyl cyclase. Neurotransmissions 2:1-4

Nathan C, Xie Q-W (1994): Nitric oxide synthases: Roles, tolls, and controls. Cell 78:915-918

Olney JW, Labruyere J, Price MT (1989): Pathological changes induced in cerebrocortical neurons by phencyclidine and related drugs. Science 244:1360-1362

Rasmussen K, Fuller RW, Stockton ME, Perry KW, Swinford RM, Ornstein PL (1991a): NMDA receptor antagonists suppress behaviors but not norepinephrine turnover or locus coeruleus unit activity induced by opiate withdrawal. Eur J Pharmacol 197:9-16

Rasmussen K, Krystal JH, Aghajanian GK (1991b): Excitatory amino acids and morphine withdrawal: Differential effects of central and peripheral kynurenic acid administration. Psychopharmacology (Berl) 105:508-512

Rees DD, Palmer RM, Schulz R, Hodson HF, Moncada S (1990): Characterization of three inhibitors of endothelial nitric oxide synthase in vitro and in vivo. Br J Pharmacol 101:746-752

Saito S, Kidd GJ, Trapp BD, Dawson T, Bredt DS, Wilson DA, Traystman RJ, Snyder SH, Hanley DF (1994): Rat spinal cord neurons contain nitric oxide synthase. Neuroscience 49:447-456

Trujillo KA, Akil H (1991a): Inhibition of morphine tolerance and dependence by the NMDA receptor antagonist MK801. Science 251:85-87

Trujillo KA, Akil H (1991b): The NMDA receptor antagonist MK-801 increases morphine catalepsy and lethality. Pharmacol Biochem Behav 38:673-675

Tseng LF, Loh HH, Wei ET (1975): Effects of clonidine on morphine withdrawal signs in the rat. Eur J Pharmacol 30:93-99

Vaupel DB, Kimes AS, London ED (1995): Comparison of 7-nitroindazole with other nitric oxide synthase inhibitors as attenuators of opioid withdrawal. Psychopharmacology (Berl) 118:361-368 